Felix Wilhelm Siebert, Michael Oehl, Hans-Rüdiger Pfister

\title{
The influence of time headway on subjective driver states in adaptive cruise control
}

Journal article | Accepted manuscript (Postprint)

This version is available at http://dx.doi.org/10.14279/depositonce-8786

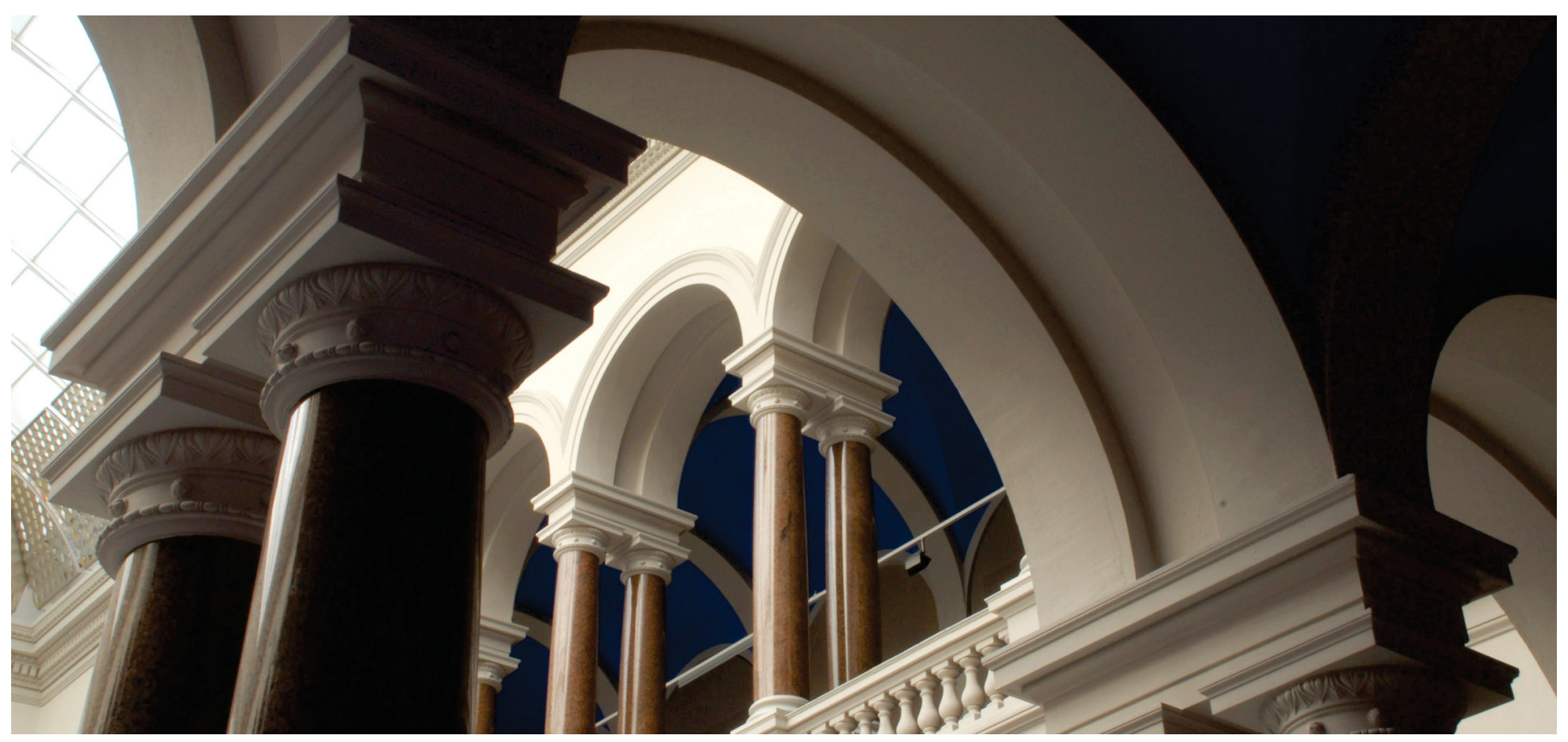

Siebert, F. W., Oehl, M., Pfister, H.-R. (2014). The influence of time headway on subjective driver states in adaptive cruise control. Transportation Research Part F: Traffic Psychology and Behaviour, 25, 65-73. https://doi.org/10.1016/j.trf.2014.05.005 
This is the Accepted Manuscript of the following article published by Elsevier in Transportation research part F: traffic psychology and behaviour [4. June 2014]:

Siebert, F. W., Oehl, M., \& Pfister, H. R. (2014). The influence of time headway on subjective driver states in adaptive cruise control. Transportation research part F: traffic psychology and behaviour, 25, 65-73. https://doi.org/10.1016/j.trf.2014.05.005

This manuscript is not the copy of record and may not exactly replicate the final, authoritative version of the article.

This work is licensed under a Creative Commons Attribution-NonCommercial-NoDerivatives 4.0 International License, http://creativecommons.org/licenses/by-nc-nd/4.0/. 
The Influence of Time Headway on Subjective Driver States in Adaptive Cruise Control

Felix Wilhelm Siebert ${ }^{\mathrm{a}}$, Michael Oehl ${ }^{\mathrm{a}}$, Hans-Rüdiger Pfister ${ }^{\mathrm{a}}$

Corresponding Author: Felix Wilhelm Siebert

anstitute of Experimental Industrial Psychology

Leuphana University of Lueneburg

Wilschenbrucher Weg 84a

21335 Lüneburg

Germany

E-mail: felix.siebert@uni.leuphana.de

E-mail: oehl@uni.leuphana.de

E-mail: pfister@uni.leuphana.de 


\begin{abstract}
There is no agreement on the relation between driving parameters and drivers' subjective states. A linear as well as a threshold relationship for different subjective variables and driving parameters has been put forward. In this study we investigate the relationship between time headway and the ratings of risk, task difficulty, effort, and comfort. Knowledge about this interrelation may advance the development of adaptive cruise control and autonomous driving and can add to the discussion about driver behavior models. An earlier study (Lewis-Evans, De Waard, \& Brookhuis, 2010) found a threshold effect for drivers' ratings of subjective variables for time headways between 0.5 and 4.0 seconds at a speed of $50 \mathrm{~km} / \mathrm{h}$. This study aims to replicate the threshold effect and to expand the findings to time headways at different speeds. A new measure for criticality was added as a categorical variable, indicating the controllability of a driving situation to give indications for the appliance of time headway in adaptive cruise control systems. Participants drove 24 short routes in a driving simulator with predefined speed and time headway to a leading vehicle. Time headway was varied eight-fold ( 0.5 to 4 seconds in 0.5 second increments) and speed was varied three-fold $(50,100,150$ $\mathrm{km} / \mathrm{h}$ ). A threshold effect for the ratings of risk, task difficulty, effort, and comfort was found for all three different speeds. Criticality proved to be a useful variable in assessing the preferred time headway of drivers.
\end{abstract}

\title{
Keywords
}

Time Headway, Adaptive Cruise Control, Driver Behavior Modeling, Criticality 


\section{Introduction}

Time headway is an important variable in the distance keeping algorithms of adaptive cruise control systems (e.g. Desjardins \& Chaib-draa, 2011; Swaroop, Hedrick, Chien, \& Ioannou, 1994; Touran, Brackstone, \& McDonald, 1999; Van Winsum, 1999). It is calculated by dividing the distance to a lead vehicle by the speed of the following vehicle, resulting in the time that it takes for the following vehicle to reach the momentary position of the lead vehicle. Since the calculation of time headway incorporates the speed of the following vehicle, it is a variable that can give valid information on distance not only for a specific velocity range, but, at least theoretically, for all possible vehicle velocities in traffic. When vehicle traffic is recorded and time headways are calculated, there is a broad range of observable time headways for self-driving. This is attributed to different traffic states. Sparse traffic for example can lead to large time headways, due to the fact that drivers can freely reach their preferred speed and the speed limit hinders close distances between vehicles, while congested roads lead to smaller time headways (Ayres, Schleuning, \& Young, 2001; Neubert, Santen, Schadschneider, \& Schreckenberg, 1999). Thus, it is hypothesized that the minimal time headway for self-driving that is considered safe by drivers can only be observed in congested traffic situations. This minimal time headway is suspected to be between 1 and 2 seconds (Ayres et al. 2001). Since in real life traffic many variables, including congestion and speed, have an effect on possible time headway ranges, attempts have been made to systematically research the influence of time headway on drivers' subjective states, such as feelings of risk, task difficulty, effort, and comfort. Therefore, the aim of this study is to systematically research the influence of time headways on the aforementioned subjective driver states. To understand the relation between this distance variable and driver states, we systematically present the driver with a set of time headways. Furthermore we aim to contribute to the development of adaptive cruise control systems and distance keeping in autonomous cars. Since small, i.e., just considered safe, time headways are more often observed in relatively low speeds due to traffic congestion (Ayres, Schleuning, \& Young, 2001), we also research if time headway is still useful as a distance variable for higher velocities, by presenting the range of time headways for 50, 100, and 150 $\mathrm{km} / \mathrm{h}$. Since time headway is a variable that is theoretically applicable for different speeds, we expect that the relation between time headway and driver states is constant over all velocities we investigate in the present study.

Apart from implications for the development of adaptive cruise control systems, this study may also add to the discussion about driver behavior modeling and the significance of different subjective variables in traffic psychology. The influence of driving parameters on drivers' subjective states has long been discussed in the traffic psychology research community (e.g. Michon, 1986; Ranney, 1994; Vaa, 2007). As of now there is no agreement on the subjective variables that determine a driver's choice of speed and safety margins or time headway to other road-users and-objects. A number of subjective variables have been researched, e.g., risk (Näätänen \& Summala, 1974; Wilde, 1982), 
comfort (Summala, 2005), effort and task difficulty (Fuller, 2005). A possible reason for the disagreement over which single subjective variable governs drivers' actions may lie in the moderate to high correlation between the aforementioned subjective variables that can be found in traffic psychology research (Lewis-Evans \& Rothengatter, 2009; Lewis-Evans et al., 2010; Fuller, 2005; Fuller, McHugh \& Pender, 2008). In light of this unresolved disagreement, it seems advisable to measure multiple subjective variables when investigating the influence of time headway on a driver's subjective state. In this study, we assess risk, task difficulty, effort, and comfort, and expect moderate to high correlations between these subjective variables.

In addition to the disagreement over which subjective variable is crucial for drivers' decision making, there is a lack of agreement over the general occurrence and awareness of subjective variables in drivers. By measuring in systematically varied driving situations in this study, we intend to advance the discussion on this topic. A general differentiation between driver behavior models is the level of awareness and occurrence of subjective variables. For example, Näätänen and Summala (1974; also Summala, 1988) argue in their zero risk model that drivers do not constantly experience risk and that there is no subconscious risk experience when driving their vehicle, but that drivers adjust safety margins while driving to avert risky situations in general. Following this theory, there is generally no occurrence or awareness of subjective risk in drivers. Risk is only infrequently experienced when drivers are pushed towards maladjusted driving behavior due to motivational factors or by actions of other road users, which is considered the exception in normal driving. Contrary to the zero risk model, Wilde (1982) argues in his theory of risk homeostasis that there is a target level of risk that is larger than zero that a driver tries to maintain by adjusting his or her subjective risk through the adjustment of driving parameters. Wilde further hypothesizes that this constant comparison is highly automatized, but can be "called into full consciousness by questioning the individual" (Wilde, 1982, p.210).

Following the zero risk theory, it would be hypothesized that a driver does not report a feeling of risk when asked to assess his subjective state in a normal self-driving condition. Following the theory of risk homeostasis, a driver would report a level of risk in a normal self-driving condition that is larger than zero. In this study, in which a driving parameter, i.e., time headway, is systematically varied, it is therefore of interest if participants report a level of risk in each time headway condition, or if participants only experience risk for certain time headways. If participants do report risk for driving conditions in which time headway is relatively large, this would support the risk homeostasis theory by Wilde (1982). If participants do not report risk for large time headways, this would add to the validity of the zero risk theory by Näätänen and Summala (1974).

There is prior research on the relation between systematically varied time headways and subjective variables. Lewis-Evans, De Waard and Brookhuis (2010) conducted a study investigating the relationship between time headway and the experience of risk, task difficulty, effort and comfort in drivers. They found a threshold effect of time headway. The characteristics of this threshold effect are 
the following: For large time headways, subjective ratings of risk, task difficulty, effort and discomfort stayed constant until a critical time headway is reached. For all time headways smaller than the threshold time headway, participants' subjective ratings of risk, task difficulty, and effort increased significantly, and ratings of comfort decreased significantly. In the study by Lewis-Evans et al. (2010), participants $(N=40)$ drove in a fixed base driving simulator with a fixed speed of $50 \mathrm{~km} / \mathrm{h}$.

Participants could not change their speed and only controlled the steering. A lead vehicle was driving ahead of participants' vehicle with an identical speed of $50 \mathrm{~km} / \mathrm{h}$. The distance of the lead vehicle was varied eight-fold, resulting in eight different time headways, ranging from 0.5 to 4.0 seconds, varied in 0.5 second increments. At the beginning of each condition, the lead vehicle and the participant's vehicle had a distance of 10 meters at a speed of $0 \mathrm{~km} / \mathrm{h}$. The speed was then increased until $50 \mathrm{~km} / \mathrm{h}$ were reached, while the distance corresponding to one of the pre-defined time headways was set during this acceleration phase. Each condition lasted approximately three minutes. In addition there was a so called free following condition, in which participants had control over their vehicle's speed and were instructed to follow the lead vehicle as close as possible while still feeling comfortable. The simulated road was designed as a typical inner city street.

After each condition in which a single pre-defined time headway was presented, participants rated task difficulty, subjective risk, effort, and comfort for the specific distance on a 7-point Likert scale. Two types of crash risk were also rated by asking participants to indicate the number of times they would lose control of the vehicle when driving with the given distance, and how often they estimated a loss of control or accident for another driver driving with the same distance in a two month period. For the non-free follow conditions, i.e., when time headways was fixed, participants also rated if they would typically follow a lead vehicle with the same distance on a 7-point scale ranging from 1 = "never" to 7 = "always". Results showed a threshold relationship between time headway and risk, task difficulty, effort and comfort, in that time headways from 4.0 to 2.0 resulted in consistently low variable ratings and increased ratings for time headways lower than 2.0 seconds.

Regression analyses supported the hypothesis of a threshold for the researched subjective variables with variable ratings showing a significant relationship with time headways of 2.0 to 0.5 seconds ( $\beta$ $=.29$ to .61 ), with no significant relation for time headways of 2.5 to 4.0 seconds ( $\beta=-.17$ to .18 ). Although the threshold point was assumed to lie between 2.0 and 1.5 seconds after descriptive analysis of the data, the split of time headways for the regression analyses was made between 2.5 and 2.0 seconds to be able to use the ratings of 2.0 seconds as the starting point for the regression line for smaller time headways. Ratings of task difficulty, feeling of risk, comfort and effort were moderately to highly correlated with each other $(r=.41$ to $.78, p<.001)$, and the mean time headway in the free follow condition was 1.78 seconds $(S D=.89)$. 
Taking the findings of Lewis-Evans et al. (2010) into account, the present study investigates if a threshold exists for time headway on a set of subjective variables for different speeds. To ensure comparability, we investigated the same subjective variables as Lewis-Evans et al. (2010) did, that is, risk, task difficulty, effort, and comfort. It is hypothesized that the threshold relationship between time headway and risk, task difficulty, effort, and comfort is consistent over different velocities. The threshold was expected to lie between 2.0 and 1.5 seconds. In line with earlier research, a high correlation between the subjective variables was anticipated (Lewis-Evans \& Rothengatter, 2009; Lewis-Evans et al., 2010; Fuller, 2005; Fuller, McHugh \& Pender, 2008).

\section{Method}

\subsection{Participants}

Participants $(N=33)$ were recruited from the student body of the Leuphana University of Lueneburg. The only prerequisite for participation was the possession of a driver's license. 16 participants were female (17 male) with a mean age of $M=22.48(S D=2.53)$. Participants owned their drivers' licenses for an average of $M=4.92(S D=2.59)$ years and had on average driven $M=56,393(S D=94,927)$ kilometers since then. On average participants drove $\mathrm{M}=7,574(S D=8,894)$ kilometers per year with $48.5 \%$ using the car at least once a week. For their participation the students were awarded with testsubject-hours which need to be collected during students' years of study.

\subsection{Materials}

A Systems Technology W500 multi-projector fixed-based driving simulator system was used in this study. The passenger cabin, dashboard, steering wheel, and gearshift were taken from an automatic Volkswagen Golf 4, a medium class vehicle. The pedal system consisted of generic driving simulator pedals. The traffic environment was projected onto three screens, each measuring 1.4 x 1.4 meters, which were positioned 2 meters away from the driver's seat. A 7-inch touchscreen was built into the center console at the height of the steering wheel. Curtains enclosed the whole simulator to shut out outside light and dampen ambient sounds. The simulator software was programmed to save data with a frequency of $20 \mathrm{~Hz}$.

Analog to the study by Lewis-Evans et al. (2010), 24 "fixed follow" situations were programmed, in which the vehicle speed and distance to another car were fixed. Each of the 24 situations paired one of three velocities (50, 100 and $150 \mathrm{~km} / \mathrm{h})$ with one of eight time headways $(0.5$ seconds to 4 seconds in 0.5 second increments). Each situation had one gentle curve, with right and left curves randomized for every situation. Combinations of time headway and velocity were presented in random order. This was 
done to counter possible adaptational effects which were found to carry over from automated driving with small time headways to self-driving (Eick \& Debus, 2005; Skottke, 2007). Each situation lasted 60 seconds and after each situation the simulator screen was blanked out. The situations were shorter than in the study by Lewis Evans et al. (2010), to keep the length of the experiment reasonable, while still being able to research all 27 conditions in one experimental treatment. Pretests showed that participants are able to rate their subjective experience for conditions of 60 seconds. In contrast to the study by Lewis-Evans et al. (2010), there was no acceleration phase, so the participant's car and the lead vehicle were driving at their specified speed from the beginning, i.e. the blank screen, to the end of each situation.

Three additional "free follow" conditions were programmed and randomly presented, in which the participants trailed a lead vehicle driving 50,100 and $150 \mathrm{~km} / \mathrm{h}$ with full control of their own car. To distinguish free follow from fixed follow conditions, a text was displayed before each free follow condition, informing participants that a free follow condition would start and reminding them to follow the lead vehicle with a distance they would maintain in real-life driving. With the instruction for participants to follow with a distance that resembles their following behavior in real-life driving we aimed for an increase of external validity for following behavior.

In the free follow conditions, the participant's vehicle drove autonomous with a fixed speed (either 50, 100 , or $150 \mathrm{~km} / \mathrm{h}$ ) following a lead vehicle with a distance equivalent of a time headway of 4.0 seconds for the first 5 seconds of the situation. After the initial autonomous phase, a short audio signal indicated that the driver now had to take over full control of the pedals and the steering wheel. Control of the vehicle was handed over to the driver 5 seconds after the audio signal. The autonomous phase was added to prevent a loss of velocity and a consequential increase in time headway due to the abrupt start of the situation, especially considering the relatively large time headway. With the help of the autonomous phase, participants had the ability to prepare their desired acceleration/deceleration activity, before taking over control of the vehicle. Every free follow condition lasted 120 seconds and had a gentle curve with left and right curves randomized. Data was recorded for the complete free follow condition, i.e. 120 seconds.

In all conditions, free and fixed follow, participants drove on a two lane road (one lane in each direction), with the lanes separated by dashed lane markings. There was no guard railing on the side of the road. To prevent overtaking, there was oncoming traffic in the opposing lane at random intervals of 5 to 15 seconds. Lane width was 3.60 meters in every situation. There were no objects on the roadside except for sparsely placed trees.

Two training conditions were programmed, one free follow and one fixed follow, to help participants get used to driving in the simulator and to make sure that participants were familiar with taking over control of the car in the free follow conditions. 
After every condition, participants answered questions (in German) on the touchscreen in the center console about their experience during the condition. In the fixed follow conditions, participants first rated task difficulty, subjective risk, effort, and comfort on a 7-point Likert scale for the distance kept by their car. Next, participants were asked if they would keep the same distance if they drove on their own, indicated on a 7-point Likert scale with the poles "never" and "very frequently". To evaluate crash risk participants were then asked how often they would lose control over the vehicle or have an accident if they would drive with the same distance every day for a period of two months. This question was also asked to evaluate the hypothetical crash risk of other drivers, by rewording the question to "How often would another driver have an accident or lose control over the car if he or she drove with the same distance for a period of two months." Both questions for crash risk (self and other) were answered on a 7-point Likert scale with the poles "never" and "very frequently".

With the last question, criticality was evaluated. Participants were first asked to give an assessment of the criticality of the distance between the two vehicles, choosing one of five categories followed by a number rating (Neukum, Lübbecke, Krüger, Mayser \& Steinle, 2008). The categories and the adjacent numbers were: "nothing noticed" (0), "harmless" (1-3), "unpleasant" (4-6), "dangerous" (7-9), and "uncontrollable" (10). Participants were able to indicate that they found a certain distance to be "unpleasant" and almost "dangerous", by choosing "unpleasant" and then " 6 ". The measure of criticality was added to have a categorically anchored question that can give real-life indications for time headways. For the free follow conditions, the questionnaire was the same, except that there was no question whether participants would keep the same distance if they drove themselves.

\subsection{Procedure}

After arriving in the driving simulator room, participants were asked to fill out a demographic questionnaire. After this, they sat down in the driver's seat of the simulator and were asked to adjust their seat, so they could sit comfortably and reached the pedals and the steering wheel. In a short instruction, participants were informed, that they were expected to drive short routes and that they would answer questions after each drive on the touchscreen in the center console. The two different types of conditions were briefly explained, as "free follow" and "fixed follow" conditions. After this, a first fixed follow training condition was started. Once finished, the researcher explained the operation of the touchscreen questionnaire. After answering the questionnaire, participants were informed about the autonomous phase of the free follow conditions and instructed to keep the same distance to the lead vehicle that they would keep in real world driving. Participants then drove the free follow condition. After answering the questionnaire for the free follow condition, participants were asked if they felt comfortable driving the simulated vehicle and if they had questions concerning the 
experiment.

Next, an individual file, containing all 27 conditions (24 fixed follow, 3 free follow) in randomized order was started. After the last condition was completed, participants received their test-subject hours and were told about the background of the experiment.

\section{Results}

To obtain a descriptive impression of the relation between systematically varied time headways and ratings of task difficulty, subjective risk, effort, comfort (recoded), typically follow (recoded), self-, and other- crash probability we plotted the mean ratings of participants (figure 1) for all eight time headways and the three velocities. It is important to keep in mind that the lowest possible rating for any subjective variable was 1 on a seven point Likert scale. Therefore, there can be no values smaller than 1. Subjective ratings of task difficulty, subjective risk, effort, comfort, as well as self- and othercrash probability stay relatively constant for time headways from 4.0 to 2.0 seconds. For time headways smaller than 2 seconds, subjective ratings increase. Descriptively, it appears that the hypothesized threshold effect can be observed. The threshold effect also appears to be consistent for all three velocities. 

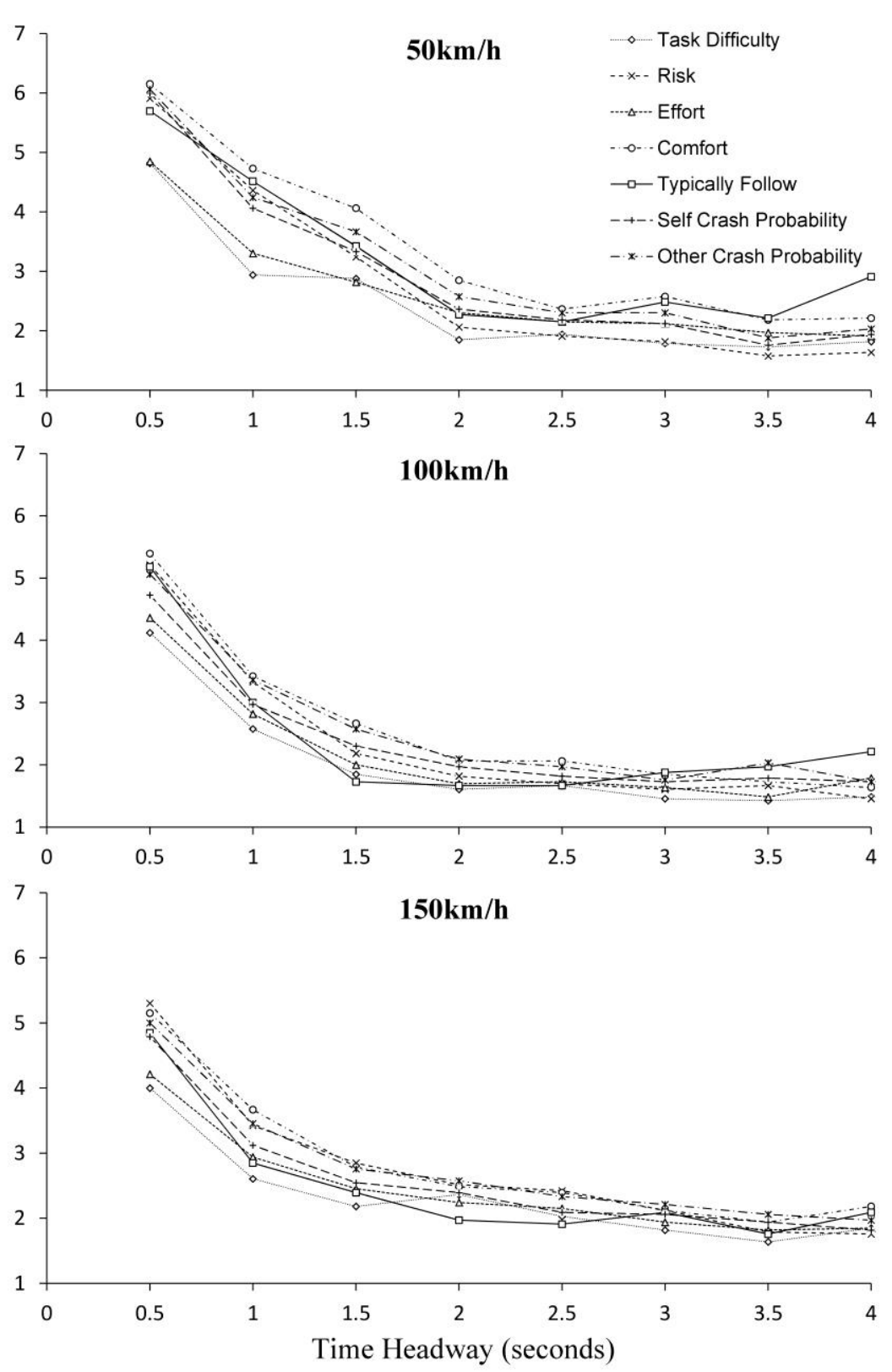

Fig. 1. Average ratings of task difficulty, subjective risk, effort, comfort, typically follow (inverted), and crash probability (self $\&$ other) for eight fixed time headways and three velocities.

A second observation can be made from the plotted data: on average the ratings of subjective variables for the large time headway conditions, i.e., 4.0 and 3.5 seconds, are very close to one. Since our goal was to add to the discussion on driver behavior models, we will go into more detail on the ratings of risk, especially for large time headways. Mean ratings of subjective risk are relatively small for time headways of 4.0 seconds for all three velocities. On average participants reported a subjective risk of $1.64(S D=0.99)$ for the $50 \mathrm{~km} / \mathrm{h}, 1.45(S D=0.91)$ for the $100 \mathrm{~km} / \mathrm{h}$, and $1.76(S D=1.12)$ for the 150 $\mathrm{km} / \mathrm{h}$ condition. The anchors on the subjective risk Likert scale were labeled "no risk" and "maximum risk". When looking at the subjective risk rating for every single participant, it becomes clear that a 
high number of participants did report "no risk" for the condition with a fixed time headway of 4.0 seconds. For the velocity of $50 \mathrm{~km} / \mathrm{h}, 21$ out of 33 participants reported to have experienced no subjective risk, for $100 \mathrm{~km} / \mathrm{h} 24$ out of 33 , and for the $150 \mathrm{~km} / \mathrm{h}$ condition with a fixed time headway of 4.0 seconds, 18 out of 33 participants indicated that they did not experience risk by marking the number 1 on the Likert scale.

Since the Likert scales used in this study were only anchored on the poles, criticality was added as a measure that is based on categories. Criticality is plotted in figure 2 for all time headways and the three researched velocities. As with the other subjective variables, it appears that there is a threshold effect of time headway and the average ratings of criticality. Criticality ratings for time headways from 4.0 to 2.0 seconds stay relatively constant, criticality then increases for subsequent smaller time headways. On average criticality is rated as "harmless" for time headways between 4.0 and 2.0 seconds. In the $50 \mathrm{~km} / \mathrm{h}$ condition the distance is then rated as unpleasant $(M=4.3, S D=1.76)$ for a time headway of 1.5 seconds. In contrast, criticality was still rated as "harmless" for a time headway of 1.5 seconds for the two higher velocities of 100 and $150 \mathrm{~km} / \mathrm{h}$. Time headways of 1.0 seconds are rated as "unpleasant" for all three velocities, and time headways of 0.5 seconds are rated as "dangerous" for all three presented speeds. In general it appears that over all time headways except the smallest ( 0.5 seconds) criticality ratings were on average the lowest for the $100 \mathrm{~km} / \mathrm{h}$ conditions.

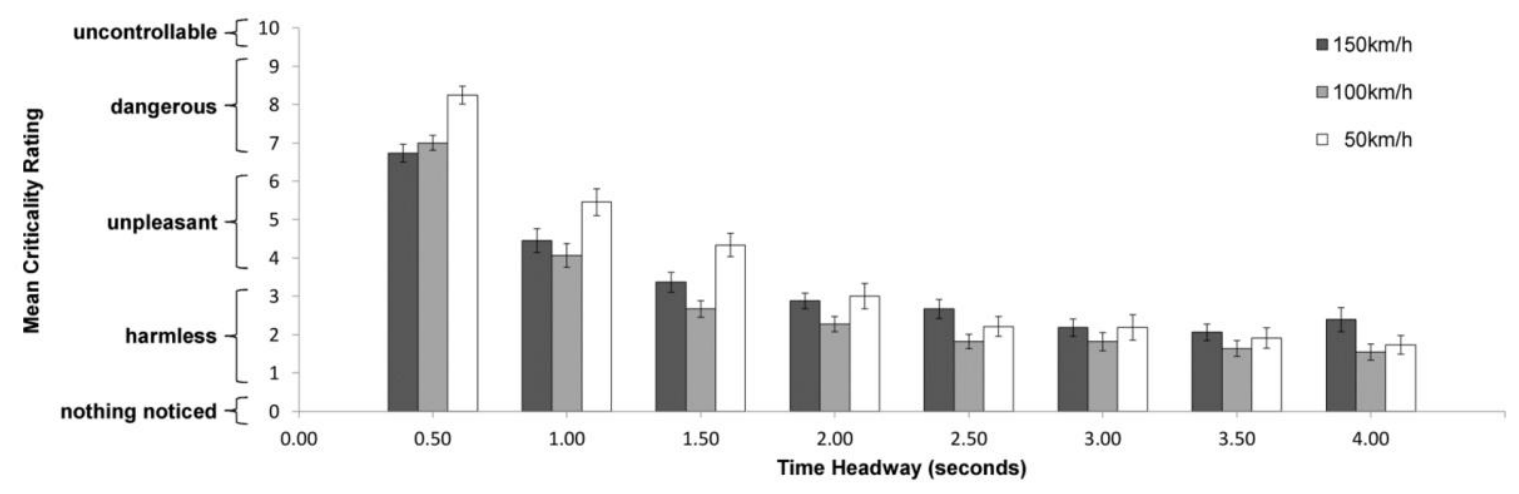

Fig. 2. Mean ratings and standard errors of criticality for eight time headways and three velocities.

Looking at figure 1 it appears that the ratings of subjective variables correlate with each other. Hence, multiple Pearson correlations were performed to analyze the relationship between the researched subjective variables. All reported subjective variables on the different time headways and speeds were significantly correlated. For the fixed follow conditions, Pearson's correlations showed that task difficulty is highly correlated with risk, effort, comfort, rating of criticality, and estimated crash risk for oneself as well as others $(r=.67$ to $.87, p<.01)$. Reported risk correlated highly with effort, comfort, rating of criticality, and crash risk for others and oneself ( $r=.80$ to $.83, p<.01)$. Effort correlated with comfort, rating of criticality, and estimated crash risk for others and oneself $(r=.67$ 
to $.73, p<.01$ ). Comfort (recoded) was highly correlated with the rating of criticality and crash risk for others and oneself $(r=.80, p<.01)$. Ratings of criticality were correlated with crash risk $(r=.79$ to $.80, p<.01)$, and crash risk for oneself correlated highly with estimated crash risk for others $(r=.95$, $p<.01)$. The indication if a participant would follow the lead vehicle with the same distance correlated negatively with the other reported subjective variables $(r=-.52$ to $-.66, p<.01)$.

After calculating the correlation between the ratings of subjective variables, a regression analysis was run separately for each subjective variable and all time headways and velocities. Taking into account the results of the study by Lewis-Evans et al. (2010) and the plotted ratings of the subjective variables and time headway presented in figure 1, the data was split between the time headways of 2.0 and 2.5 seconds. Multiple regression analyses were then run for subjective ratings of time headways between 4.0 and 2.5 seconds, and subjective ratings of time headways between 2.0 and 0.5 seconds. Regression analyses were also run over all three velocities of 50,100 , and $150 \mathrm{~km} / \mathrm{h}$.

The results of these multiple regression analyses are presented in table 1. Regression analyses revealed no significant relation between time headways of 4.0 to 2.5 seconds and the subjective ratings of participants. This supports the descriptive findings of the plotted data (Fig. 1) and the results of LewisEvans et al. (2010). Time headways ranging from 4.0 to 2.5 seconds do not significantly influence the ratings of task difficulty, risk, effort, comfort, criticality, and estimated self- and other-crash risk. This result was consistent for all the velocities. There was one exception: Ratings of subjective risk showed a significant relation to time headways from 4.0 to 2.5 seconds for a speed of $150 \mathrm{~km} / \mathrm{h}$. It is important to recognize that the regression model can only explain $5 \%\left(\mathrm{R}^{2}=.05\right)$ of the variance in the ratings of subjective risk for a velocity of $150 \mathrm{~km} / \mathrm{h}$.

For time headways ranging from 2.0 to 0.5 seconds a significant $(\mathrm{p}<.001)$ linear relation between time headways and all ratings of subjective variables was found. Overall, the regression model can explain a moderate to high percentage of variance in the subjective ratings of participants for time headways of 2.0 to 0.5 seconds. This result was also consistent over all the researched velocities. 
Table 1

Regression analyses for ratings of task difficulty, subjective risk, effort, comfort (recoded), crash probability (self \& other), typically follow (not recoded), and criticality, for time headways from 4.0 to 2.5 , and from 2.0 to 0.5 seconds.

Time Headway $=4.0$ to 2.5

Time Headway $=2.0$ to 0.5

\begin{tabular}{|c|c|c|c|c|c|c|}
\hline \multicolumn{7}{|l|}{ Speed $=50 \mathrm{~km} / \mathrm{h}$} \\
\hline & $\mathrm{R}^{2}$ & Beta & $\mathrm{t}$ & $\mathrm{R}^{2}$ & Beta & $\mathrm{t}$ \\
\hline Task Difficulty & 0.00 & -0.04 & -0.43 & $0.30^{* * *}$ & -0.55 & -7.47 \\
\hline Subjective Risk & 0.01 & -0.11 & -1.27 & $0.52^{* * *}$ & -0.72 & -11.79 \\
\hline Effort & 0.01 & -0.07 & -0.83 & $0.26^{* * *}$ & -0.51 & -6.82 \\
\hline Comfort & 0.01 & -0.07 & -0.79 & $0.46^{* * *}$ & -0.68 & -10.59 \\
\hline Self Crash Probability & 0.01 & -0.11 & -1.26 & $0.45^{* * *}$ & -0.67 & -10.29 \\
\hline Other Crash Probability & 0.02 & -0.12 & -1.42 & $0.40^{* * *}$ & -0.63 & -9.32 \\
\hline Typically Follow & 0.02 & -0.13 & -1.53 & $0.51^{* * *}$ & 0.71 & 11.54 \\
\hline Criticality Rating & 0.02 & -0.12 & -1.41 & $0.37^{* * *}$ & -0.61 & -8.67 \\
\hline \multicolumn{7}{|l|}{ Speed $=100 \mathrm{~km} / \mathrm{h}$} \\
\hline & $\mathrm{R}^{2}$ & Beta & $\mathrm{t}$ & $\mathrm{R}^{2}$ & Beta & $\mathrm{t}$ \\
\hline Task Difficulty & 0.01 & -0.08 & -0.93 & $0.35^{* * *}$ & -0.59 & -8.29 \\
\hline Subjective Risk & 0.01 & -0.08 & -0.91 & $0.51^{* * *}$ & -0.71 & -11.63 \\
\hline Effort & 0.00 & 0.00 & 0.05 & $0.37^{* * *}$ & -0.61 & -8.82 \\
\hline Comfort & 0.02 & -0.15 & -1.74 & $0.52^{* * *}$ & -0.72 & -11.80 \\
\hline Self Crash Probability & 0.00 & -0.03 & -0.35 & $0.41^{* * *}$ & -0.64 & -9.52 \\
\hline Other Crash Probability & 0.00 & -0.06 & -0.72 & $0.46^{* * *}$ & -0.68 & -10.44 \\
\hline Typically Follow & 0.01 & -0.12 & -1.32 & $0.51^{* * *}$ & 0.72 & 11.72 \\
\hline Criticality Rating & 0.01 & -0.09 & -1.07 & $0.58^{* * *}$ & -0.76 & -13.50 \\
\hline
\end{tabular}

Speed $=150 \mathrm{~km} / \mathrm{h}$

\begin{tabular}{lcccccr}
\hline & $\mathrm{R}^{2}$ & Beta & $\mathrm{t}$ & $\mathrm{R}^{2}$ & Beta & $\mathrm{t}$ \\
\cline { 2 - 7 } Task Difficulty & 0.01 & -0.07 & -0.82 & $0.19^{* * *}$ & -0.44 & -5.51 \\
Subjective Risk & $0.05^{*}$ & -0.22 & -2.56 & $0.37^{* * *}$ & -0.60 & -8.65 \\
Effort & 0.01 & -0.11 & -1.25 & $0.25^{* * *}$ & -0.50 & -6.60 \\
Comfort & 0.01 & -0.08 & -0.86 & $0.39^{* * *}$ & -0.63 & -9.13 \\
Self Crash Probability & 0.02 & -0.12 & -1.41 & $0.33^{* * *}$ & -0.58 & -8.02 \\
Other Crash Probability & 0.02 & -0.15 & -1.72 & $0.35^{* * *}$ & -0.59 & -8.34 \\
Typically Follow & 0.00 & -0.02 & -0.18 & $0.36^{* * *}$ & 0.60 & 8.60 \\
Criticality Rating & 0.01 & -0.07 & -0.83 & $0.47^{* * *}$ & -0.69 & -10.71 \\
\hline
\end{tabular}

$* \mathrm{p}<0.05 * * * \mathrm{p}<0.001$.

Time headway for self-driving was calculated for the last 30 seconds of the two minute free follow conditions. Mean time headways were $M_{50 \mathrm{~km} / \mathrm{h}}=3.06$ seconds $(S D=0.85)$ for the $50 \mathrm{~km} / \mathrm{h}$ condition, $M_{100 \mathrm{~km} / \mathrm{h}}=2.96$ seconds $(S D=1.20)$ for the $100 \mathrm{~km} / \mathrm{h}$ condition, and $M_{150 \mathrm{~km} / \mathrm{h}}=3.97$ seconds $(S D=$ 2.88) for the condition in which the lead vehicle was driving at a speed of $150 \mathrm{~km} / \mathrm{h}$. Compared to the 
results of Lewis-Evans et al. (2010) the time headways in the free following conditions of this study were higher.

\section{Discussion}

In this study we investigated the relationship between time headway as a variable for distance calculation of adaptive cruise control and a set of subjective variables. Time headway was systematically varied to investigate the type of relation between time headways and subjective driver states. Additionally, time headways were presented for three different velocities to research if the type of relationship between time headway and subjective variables was constant over different velocities. In line with earlier research, we expected a high correlation between the subjective ratings given by participants. To add to the ongoing discussion on driver behavior models, we analyzed the general occurrence of subjective risk in participants. Furthermore we introduced a measurement of criticality as a categorical rating. Generally, we wanted to contribute to the development of vehicle automation, i.e. adaptive cruise control and autonomous driving.

The main objective of this study was to research the interrelation of time headway and risk, task difficulty, effort, and comfort. Regression analyses support the notion of a general threshold of time headway and subjective variables for $50 \mathrm{~km} / \mathrm{h}$. This supports the results of Lewis-Evans et al. (2010). The threshold relationship was also found to be consistent over all three researched velocities. Subsequent time headways ranging from 4.0 to 2.5 seconds did not significantly influence the subjective ratings of drivers. For time headways ranging from 2.0 to 0.5 seconds, the regression analysis revealed a significant influence of subsequent time headways on the subjective variables. These results indicate that the critical time headway lies between 2.0 and 1.5 seconds. Based on these results, it appears that time headway can provide a good indication for preferred distances in vehicle traffic for different velocities.

Our second hypothesis was that all subjective variables that were measured correlate significantly with each other. The results showed that this is the case. We found moderate to high correlations between all ratings given by participants. Although this result does not help to resolve the disagreement over which single subjective variable is crucial to determine a driver's behavior when controlling a vehicle, it supports earlier findings on drivers' internal affective state (Lewis-Evans \& Rothengatter, 2009; Lewis-Evans et al., 2010; Fuller, 2005; Fuller, McHugh \& Pender, 2008). The moderate to high correlations appear plausible. A high task difficulty in a given driving situation is likely to accompany a high effort, diminishing comfort and an increased subjective and crash risk. Furthermore, it can be assumed that a driver will not typically follow another vehicle with a distance that is demanding a high level of effort and task difficulty, excites a feeling of subjective risk and crash risk, and is uncomfortable. 
A further objective of this study was to add to the discussion about the general occurrence of subjective variables in drivers. While results of a simulator study cannot substitute for research in real traffic, our results show that a majority of drivers in our sample did not report subjective risk for a number of time headways when driving in the simulator. This is in line with the zero risk model by Näätänen and Summala (1974). For the largest time headway of 4.0 seconds, participants indicated that they would typically follow with the equivalent distance, but the majority did not report a subjective feeling of risk. Further research needs to be conducted to explore if these results remain the same in real life driving.

The specific type of interrelation between time headways and subjective variables, named threshold effect in this study, hints to the importance of the adjustment of distance parameters in adaptive cruise control (ACC) and autonomous driving. If even a small gradual change in time headway can lead to a substantial change in drivers' subjective appraisal of a driving situation when time headway falls below the threshold, it is crucial for developers of ACC systems and autonomous vehicles to keep time headways over this threshold. It appears that time headways higher than the threshold do not result in a negative subjective experience for drivers. Large time headways were rated high on comfort, allowing car system developers to utilize a distance of the equivalent of up to 2.0 seconds as a comfort buffer. It is important to keep in mind that time headway is just one of several variables that can be computed for vehicle following. If developers of adaptive cruise control systems decide to use time headway as a variable for distance calculation, it appears advisable to keep the threshold effect in mind.

The measurement of criticality proved to be useful to get an impression of drivers' subjective states. Mean criticality for $50 \mathrm{~km} / \mathrm{h}$ was rated as unpleasant for time headways lower than 2.0 seconds. For higher speeds, criticality was on average rated as unpleasant for time headways lower than 1.5 seconds. Figure 2 also shows that criticality was rated higher for time headways between 0.5 and 2.0 seconds for the $50 \mathrm{~km} / \mathrm{h}$ conditions than for the two higher velocities of 100 and $150 \mathrm{~km} / \mathrm{h}$. In general, criticality ratings of the $100 \mathrm{~km} / \mathrm{h}$ conditions were rated lower than for conditions of 50 and $150 \mathrm{~km} / \mathrm{h}$ conditions, except for the smallest time headway of 0.5 seconds. A possible explanation for this effect could be the roadside design of the simulation. While the lane width that was chosen for this study resembles a highway, the roadside in the simulation had only sparsely placed trees and no guard railing. This design might bear a resemblance to a German country road. Country roads in Germany have a general speed limit of $100 \mathrm{~km} / \mathrm{h}$; speeds of $50 \mathrm{~km} / \mathrm{h}$ might have been rated as more critical by participants because they are perceived to be too slow for a country road, while speeds of $150 \mathrm{~km} / \mathrm{h}$ are perceived as being "over the speed limit" for country roads. Looking at the larger time headways in this study, large time headways and resulting high distances to leading vehicles are not rated as critical. This supports the results for ratings of comfort discussed earlier.

The free follow time headways were almost twice as high in this study, compared to earlier results by Lewis-Evans et al. (2010). This shows the importance of participants' instruction for the free follow 
condition. While Lewis-Evans et al. instructed participants to follow as close as possible while still feeling comfortable, participants in this study were instructed to follow with the same distance they would keep in real life driving. Since this lead to large time headways, it appears advisable to instruct participants to follow as close as possible while still feeling comfortable.

There are some limitations to the results of this study. It is important to keep in mind that there were no motivational factors induced in participants in this study. It is plausible that drivers would follow with small time headways in real life driving once motivational factors emerge, even though they rate them high on risk etc. and indicate that they would not typically follow this close in this study. This might be the reason for the relatively high time headways in the free follow conditions. These large time headways are not close to the threshold time headway. Furthermore, the duration of free follow conditions was fixed and participants were not allowed to pass the lead vehicle. This might have further inhibited participants to advance the lead vehicle, since there was no time benefit in a small distance to the lead vehicle. Another limitation of this study is the presentation of the questions for subjective ratings. The number of questions that participants had to answer after every condition might have led to response patterns. This possibility has to be kept in mind, especially when assessing the moderate to high correlation between subjective ratings. Although several questions were recoded, parts of the correlation might be explainable through these response patterns. As reported earlier, there might have been interference in the results for criticality due to the roadway design. To counter this interference it seems advisable to design the roadway compatible with expected speeds. The roadway design of the $50 \mathrm{~km} / \mathrm{h}$ conditions should therefore be modeled after inner city streets, the $100 \mathrm{~km} / \mathrm{h}$ condition after country roads, and the $150 \mathrm{~km} / \mathrm{h}$ condition roadway should be designed like a highway.

With the present study we advanced the current body of literature on the relation of time headway, and subjective driver states. In particular we successfully replicated past research indicating a threshold effect for time headway and risk, task difficulty, effort, and comfort. Moreover, we extended this finding to different velocities. This is important for the design of driver assistant systems and automated driving. We hope that our results can help to build comfortable solutions for distance keeping in adaptive cruise control systems and the acceptance of autonomous vehicles. 


\section{References}

Ayres, T. J., Li, L., Schleuning, D., \& Young, D. (2001). Preferred time-headway of highway drivers. In Intelligent Transportation Systems, 2001. Proceedings. 2001 IEEE (pp. 826-829). IEEE.

Desjardins, C., \& Chaib-draa, B. (2011). Cooperative adaptive cruise control: A reinforcement learning approach. Intelligent Transportation Systems, IEEE Transactions on, 12(4), 1248-1260.

Eick, E.-M. \& Debus, G. (2005). Adaptation effects in an automated car-following scenario. In G. Underwood (Ed.), Traffic \& Transport Psychology. Theory and Application (pp. 243-255). Amsterdam: Elsevier.

Fuller, R. (2005). Towards a general theory of driver behaviour. Accident Analysis \& Prevention, 37(3), 461-472.

Fuller, R., McHugh, C., \& Pender, S. (2008). Task difficulty and risk in the determination of driver behaviour. Revue Européenne de Psychologie Appliquée/European Review of Applied Psychology, 58(1), 13-21.

Lewis-Evans, B., De Waard, D., \& Brookhuis, K. A. (2010). That's close enough-A threshold effect of time headway on the experience of risk, task difficulty, effort, and comfort. Accident Analysis \& Prevention, 42(6), 1926-1933.

Lewis-Evans, B., \& Rothengatter, T. (2009). Task difficulty, risk, effort and comfort in a simulated driving task-Implications for Risk Allostasis Theory. Accident Analysis \& Prevention, 41(5), 1053-1063.

Michon, J. A. (1986). A critical view of driver behavior models: what do we know, what should we do? In: Evans, L. \& Schwing, R.C. (Eds.), Human behavior and traffic safety (pp. 485-524). Springer US.

Näätänen, R., \& Summala, H. (1974). A model for the role of motivational factors in drivers' decisionmaking. Accident Analysis \& Prevention, 6(3), 243-261.

Neubert, L., Santen, L., Schadschneider, A., \& Schreckenberg, M. (1999). Single-vehicle data of highway traffic: A statistical analysis. Physical Review E,60(6), 6480.

Neukum, A., Lübbecke, T., Krüger, H.-P., Mayser, C., Steinle, J. (2008). ACC-Stop\&Go: Fahrverhalten an funktionalen Systemgrenzen. In Maurer, M. \& Stiller, C. (Eds.), 5. Workshop Fahrerassistenzsysteme (FAS), 141-150.

Ranney, T. A. (1994). Models of driving behavior: a review of their evolution. Accident Analysis \& Prevention, 26(6), 733-750.

Skottke, E.-M. (2007). Automatisierter Kolonnenverkehr und adaptiertes Fahrverhalten [Automated car following and driving behavior adaptation]. Hamburg, Germany: Verlag Dr. Kovač.

Summala, H. (1988). Risk control is not risk adjustment: The zero-risk theory of driver behaviour and its implications. Ergonomics, 31(4), 491-506.

Summala, H. (2005). Traffic psychology theories: towards understanding driving behaviour and safety efforts. In G. Underwood (Ed.), Traffic \& Transport Psychology (pp. 383-394). Oxford, UK: Elsevier.

Swaroop, D. V. A. H. G., Hedrick, J. K., Chien, C. C., \& Ioannou, P. (1994). A Comparision of Spacing and Headway Control Laws for Automatically Controlled Vehicles1. Vehicle System Dynamics, 23(1), 597-625.

Touran, A., Brackstone, M. A., \& McDonald, M. (1999). A collision model for safety evaluation of autonomous intelligent cruise control. Accident Analysis \& Prevention, 31(5), 567-578.

Vaa, T. (2007). Modelling driver behaviour on basis of emotions and feelings: intelligent transport systems and behavioural adaptations. In Modelling driver behaviour in automotive environments (pp. 208-232). Springer London. 
Van Winsum, W. (1999). The human element in car following models. Transportation research part F: traffic psychology and behaviour, 2(4), 207-211.

Wilde, G. J. (1982). The theory of risk homeostasis: implications for safety and health. Risk analysis, 2(4), 209225 . 